\section{SKIN RELATED COMPLICATIONS IN CHILDREN TREATED WITH HIP SPICA CASTS FOR FEMUR FRACTURES}

\author{
R.L. DiFazio', J.A. Vessey², D. Zurakowski ${ }^{3}$, \\ T. Matheney
}

${ }^{1}$ Department of Orthopaedics, Children's Hospital, Boston, ${ }^{2}$ Professor, Boston College, Chestnut Hill,

${ }^{3}$ Department of Biostatistics, Chldren's Hospital, Boston, MA, USA

Purpose: Document the incidence of skin related complications in children treated with a spica cast for femur fractures and determine associated cast change charges.

Background: Closed reduction and spica casting remains the treatment of choice for femur fractures in children $<6$ years. Caring for a child in a hip spica cast poses challenges for caregivers including keeping the cast clean. Skin breakdown caused by moisture causes discomfort and the need for repeat casting. Skin related complications are financially costly to both the family and the healthcare system. The incidence of skin related complications is poorly documented.

Methods: A retrospective chart review (2003-2009) was conducted on children who sustained a femur fracture and who were treated with spica casting. Skin related complications were identified and costs associated with a cast change were calculated.

Results: The study included 300 cast placements. Two hundred and twenty three completed spica cast treatment without any reported skin complications. Seventy seven of the children had skin problems with 24 of these requiring a cast change in the OR, 34 requiring early bi-valving and lining of the cast and, and 19 requiring additional skin care. The median costs for a cast change was $\$ 14,731.00$

Conclusion: Spica cast treatment is associated with numerous skin related problems and additional costs. These data will serve as baseline data of complications secondary to spica casting for femur fractures and are benchmarks for quality improvement programs as well as alternative treatment options such as Gortex cast padding or internal fixation.

\section{NONMEDICAL OUT-OF-POCKET EXPENSES: A HIDDEN CONSEQUENCE OF HOSPITALIZATION}

\author{
J.A. Vessey ${ }^{1}$, R. Difazio ${ }^{2}$
}

${ }^{1}$ Nursing, Boston College, Chestnut Hill, ${ }^{2}$ Department of Orthopaedics, Children's Hospital, Boston, Boston, MA, USA

Purpose: To calculate the nonmedical out-of-pocket expenses (NOOPEs) incurred by families during the hospitalization of their child following orthopaedic surgical interventions.

Background: In today's healthcare climate, increased attention is being paid to the availability, affordability, and adequacy of healthcare insurance needed to cover necessary healthcare expenditures. Virtually, no public or private health plan covers the entire array of health care costs. Little attention has been placed on the magnitude NOOPES to families. NOOPEs include costs that are directly attributed to the family's health care needs including travel, parental food and lodging, dependent care, diminished parental wages, and incidental expenses. Expenses exeeding 10\% of a family's income are considered to constitute family burden. Although NOOPEs are incurred by virtually every family with a hospitalized child, there virtually no information specifically addresses the financial and psychological impact of NOOPEs on family life.

Methods: This study uses a prospective, mixed method design. The sampling frame consists families $(\mathrm{N}=32)$ whose children are hospitalized for orthopaedic surgeries and is stratified $(2 \times 2)$ by admission type (planned or unplanned) and location of family's home (local or distant) to the facility. Families complete an online expense diary to capture the NOOPEs they have incurred. Interviews of parents conducted upon discharge further illuminate diary data. Descriptive statistics and ANOVAs for key variables are calculated. Content analysis of interview data identifies common themes related to family life, stressors, and coping strategies.

Results: Pending. 